\title{
Highlights from the British Society for Heart Failure 20th Annual Autumn Meeting: three decades of heart failure
}

\author{
Alexandra Al Abel*,1 \\ ${ }^{1}$ Hull York Medical School, Hull, HU6 7RX, UK \\ *Author for correspondence: alexandra.abel@hyms.ac.uk
}

The 20th Annual Autumn Meeting of the British Society for Heart Failure took place on the 23-24 November 2017 at the Queen Elizabeth II Conference Centre, London, UK. Over 800 delegates were in attendance: a multidisciplinary league of professionals who treat patients with heart failure, including specialist nurses, trainees, cardiologists, geriatricians, pharmacists and general practitioners. The theme of the conference was 'three decades of heart failure' and celebrated the success of modern heart failure management. This report highlights the 'three decades' session, the clinical trials update, and the main discussion points from heart failure question time.

First draft submitted: 24 January 2018; Accepted for publication: 9 February 2018; Published online: 16 May 2018

Keywords: atrial fibrillation $\bullet$ British Society for Heart Failure $\bullet$ clinical trials $\bullet$ heart failure $\bullet$ heart failure with preserved ejection fraction • heart failure with reduced ejection fraction • HeFPEF • HrFREF • sacubitril-valsartan

\section{Three decades of heart failure}

The conference celebrated 30 years since the publication of CONSENSUS, the first study to show mortality benefit of a drug for heart failure (enalapril) [1]. Aptly, the eminent lead author of CONSENSUS, Karl Swedberg (University of Gothenburg, Sweden), delivered the Philip Poole-Wilson Memorial Lecture on neuro-hormonal antagonism in left ventricular systolic dysfunction (LVSD). It was not until 27 years after CONSENSUS that enalapril was finally bested by a new drug: sacubitril-valsartan in PARADIGM-HF [2]. Sacubitril-valsartan, an angiotensin receptor neprilysin inhibitor (ARNI), is now recommended by NICE for patients with symptomatic chronic heart failure with reduced ejection fraction (HeFREF) swapping from an ACE inhibitor (ACEI) or angiotensin receptor blocker. Summarizing three decades of research, Swedberg stated it is clear that patients with LVSD need to be on a combination of three neuro-hormonal blockers: ACEI/ARNI, $\beta$-blocker, and mineralocorticoid receptor antagonist.

Theresa McDonagh (King's College Hospital, London, UK) outlined the rise of natriuretic peptides as biomarkers in heart failure. B-type natriuretic peptide (BNP) and its N-terminal associate (NT-proBNP) show excellent sensitivity as biomarkers, and NICE guidelines recommend BNP or NT-proBNP in the diagnostic pathway for patients who present with clinical features of heart failure.

John Cleland (University of Glasgow, UK) gave the conference a short history of cardiac resynchronization therapy, which can improve morbidity and mortality in selected patients. He emphasized that increasing QRS duration remains the only robust predictor of a beneficial response to cardiac resynchronization therapy. Cleland stated that around $10 \%$ of patients without left bundle branch block develop longer QRS complexes per year and recommended an annual ECG (which also monitors for atrial fibrillation). 


\section{Devices \& surgical intervention}

The DANISH study previously concluded no overall survival benefit for implantable cardioverter defibrillators in patients with nonischemic cardiomyopathy [3]. Further analysis suggested mortality benefit for younger patients, but beyond around 70 years of age, there was no improvement in all-cause mortality [4]. There is a similar age interaction in the STICHES study, which found a modest overall survival benefit for coronary artery bypass grafting in patients with heart failure and severe coronary artery disease [5]. McMurray said it is likely that as people get older, comorbidities kick in and patients begin to die from causes other than heart failure; therefore, it becomes difficult to reduce mortality with interventions such as implantable cardioverter defibrillators or coronary artery bypass grafting.

Some patients with advanced heart failure require left ventricular assist devices (LVAD) and cardiac transplantation. Two studies tested newer LVADs. In MOMENTUM3, the Heartmate III device showed some advantage over the Heartmate II [6]. In ENDURANCE, the HeartWare device was deemed noninferior to the Heartmate II [7]. This year marked the 50th anniversary of the first heart transplant and McMurray noted some interesting science regarding xenotransplantation: using a new gene editing technology, it might be possible to remove the endogenous retroviruses that affect fetal pigs [8]. There is potential to increase organs available for transplant without the risk of transmitting viruses across species.

\section{Acute heart failure \& HeFPEF}

New treatment strategies for acute heart failure have long been elusive, and recent drug trials with endogenous peptides (ularitide in TRUE-AHF [9] and serelaxin in RELAX-AHF 2 [10]) were neutral. Furthermore, GUIDE-IT, a trial investigating NT-proBNP guided treatment in acute heart failure, was stopped for futility as it was no more effective than standard care [11].

Unfortunately, we still have no evidence-based treatments for patients with heart failure with preserved ejection fraction (HeFPEF). The EDIFY pilot study showed no benefit of ivabradine in HeFPEF [12]. However, in the Phase II trial REDUCE LAP-HF I, patients who had an interatrial shunt device compared with sham control saw a reduction in left atrial pressure [13]: further evaluation will determine if this effect could confer clinical benefit. Meanwhile, the Phase III morbidity/mortality trial PARAGON-HF is underway for sacubitril-valsartan in HeFPEF.

McMurray said that HeFPEF remains a poorly understood phenomenon, but he thought the populations were probably no more heterogenous than those in HeFREF trials. However, he noted an important consideration in contemporary HeFPEF trials is strict inclusion criteria to make sure that patients who do not have heart failure are not included in trials.

\section{Prevention \& other trials}

Heart failure prevention is an increasingly interesting area of research. Trials in patients with Type 2 diabetes using SGTL2 inhibitors (empagliflozin in EMPA-REG [14] and canagliflozin in CANVAS [15]) have shown reduction in cardiovascular mortality, lower heart failure hospitalization, and a reduced rate of decline in renal function. These encouraging outcomes raise the questions of whether these drugs are beneficial for all heart failure patients, or only those with diabetes, and can they be used to treat established heart failure? Further investigation is underway with Phase III morbidity/mortality trials in both HeFREF (Dapa-HF and EMPEROR-reduced) and HeFPEF (EMPEROR-preserved).

Other new trials reported include CASTLE-AF (atrial fibrillation and heart failure), which found catheter ablation of atrial fibrillation reduced all-cause mortality and mitigated worsening heart failure compared with conventional pharmacological therapy [16]. Lastly, good news for coffee connoisseurs: even very high doses of caffeine do not cause atrial or ventricular arrhythmias [17].

\section{Heart failure question time}

Question time invited audience participation and promoted topical debate. The session was chaired by Andrew Clark (University of Hull, UK) with a panel composed of John McMurray, Paul Kalra (Portsmouth Hospitals NHS Trust, UK), Annie MacCallum (Gloucestershire Care Services NHS Trust, UK), and Nigel Rowell (GPSI Cardiology, James Cook University Hospital, Middlesborough, UK).

The opening question related to heart failure with 'mid-range' ejection fraction (HeFMREF). Is HeFMREF a useful definition? Should these patients be treated with the full force of neuro-hormonal blockers? McMurray 
cautioned that with such a narrow range for ejection fraction, it is almost impossible to define these patients. The standard deviation around an ejection fraction measurement is approximately $7 \%$, so the range we are looking at is about the same as the error around the measurement. McMurray noted that evidence indicates that these patients respond to interventions and Kalra added that, in clinical practice, these patients are commonly considered to have 'mild LVSD' and are treated in the same way as patients with HeFREF.

A number of questions expressed concern over inappropriate roles for heart failure specialist nurses. MacCallum explained that many heart failure specialist nurses have been asked to become 'generic specialists' and to fill rota gaps. She emphasized the importance of collecting data on nurse-associated outcomes to provide evidence to managers on the importance of the specialist nurse role. MacCallum also recommended accessing support from the new British Society for Heart (BSH) Failure Nurse Forum. Rowell stated he thought there should be core modules on heart failure for General Practitioners, but the remit is simply too large for general practice: we need specialist nurses who are used to caring for these complex patients.

The panel then broached an ethical question: is it enough to demonstrate symptomatic benefit in a trial before initiating new treatments, or should we wait for definitive evidence on mortality? Kalra indicated that he thought it was unethical to proceed without the definitive data, as we have seen interventions that seem to improve symptoms, but which are associated with harm in definitive studies. However, Rowell emphasized that symptomatic benefit should not be forgotten and is important both for patients' quality of life and the demands they make on social care and other services.

Introducing interventions into clinical practice can be tricky, and a question was raised about starting sacubitrilvalsartan: if a patient on full dose ACEI only manages to get onto the smallest dose of ARNI, would it be better to switch them back? McMurray explained that case series suggest approximately $50-80 \%$ of people can be uptitrated to the target dose of sacubitril-valsartan $(97 \mathrm{mg} / 103 \mathrm{mg}$ b.d.), but that below $49 \mathrm{mg} / 51 \mathrm{mg}$ b.d., patients are unlikely to benefit. However, he noted there are often other blood pressure lowering drugs that could be reduced or discontinued to allow successful up-titration, including nitrates and calcium channel blockers. He stated a thorough patient assessment is essential before switching, and if a patient appears euvolemic, you might consider reducing the loop diuretic. The difficulty with switching is that patients need to be monitored very carefully. One might consider whether in the near future it will be common practice for patients to be started on ARNI instead of ACEI.

\section{Other talks in brief}

The BSH Research Fellow Simon Beggs (University of Glasgow, UK) presented an update on his RHYTHM-HF study, which uses an implantable loop recorder to examine the role of arrhythmias in heart failure. Funding from the British Heart Foundation has expanded the scope of the project to include novel substudies, such as 'why do patients with heart failure die?'.

The BSH Young Investigator's Award 2017 was won by Nathalie Conrad (University of Oxford, UK) for her work on temporal trends and patterns in heart failure incidence in the UK. Conrad's research found that the burden of heart failure is now similar to the four most common cancers combined, with a significant socioeconomic predictive component [18].

Other topics presented at the meeting include peripartum cardiomyopathy, cardio-oncology, cardiac transplantation and LVAD technologies, modern management of the right heart, sports and exercise in heart failure, outpatient-based therapies, strategies in specialist service provision and interesting case presentations. A full report program, report and details of support for the meeting are available on the BSH website.

Future BSH meeting: 21st Annual Autumn Meeting, Queen Elizabeth II Centre, London, 29-30 November 2018. Website: www.bsh.org.uk

Financial \& competing interests disclosure

The author has no relevant affiliations or financial involvement with any organization or entity with a financial interest in or financial conflict with the subject matter or materials discussed in the manuscript. This includes employment, consultancies, honoraria, stock ownership or options, expert testimony, grants or patents received or pending, or royalties.

No writing assistance was utilized in the production of this manuscript. 


\section{References}

1. The CONSENSUS Trial Study Group. Effects of enalapril on mortality in severe congestive heart failure. Results of the Cooperative North Scandinavian Enalapril Survival Study (CONSENSUS). N. Engl. J. Med. 316(23) 1429-1435 (1987).

2. McMurray JJ, Packer M, Desai AS et al. Angiotensin-neprilysin inhibition versus enalapril in heart failure. N. Engl. J. Med. 371(11), 993-1004 (2014).

3. Kober L, Thune JJ, Nielson JC et al. Defibrillator implantation in patients with nonischaemic systolic heart failure. N. Engl. J. Med. 375(13), 1221-1230 (2016).

4. Elming MB, Nielson JC, Videbaek L et al. Age and outcomes of primary prevention implantable cardioverter-defibrillators in patients with nonischemic systolic heart failure. Circulation 136(19), 1772-1780 (2017).

5. Petrie MC, Jhund PS, She L et al. Ten-year outcomes after coronary artery bypass grafting according to age in patients with heart failure and left ventricular systolic dysfunction. Circulation 134(18), 1314-1324 (2016).

6. Mehra MR, Naka Y, Uriel N et al. A fully magnetically levitated circulatory pump for advanced heart failure. N. Engl. J. Med. 376(5), 440-450 (2017).

7. Rogers JG, Pagani FD, Tatooles AJ et al. Intrapericardial left ventricular assist device for advanced heart failure. N. Engl. J. Med. 376(5), 451-460 (2017).

8. Denner J. Paving the path toward porcine organs for transplantation. N. Engl. J. Med. 377(19), 1891-1893 (2017).

9. Packer M, O'Connor C, McMurray JJV et al. Effect of ularitide on cardiovascular mortality in acute heart failure. N. Engl. J. Med. 376(20), 1956-1964 (2017).

10. Efficacy, safety and tolerability of serelaxin when added to standard therapy in AHF (RELAX-AHF 2). ClinicalTrials.gov Identifier: NCT01870778. https://clinicaltrials.gov/ct2/show/NCT01870778

11. Felker GM, Anstrom KJ, Adams KJ et al. Effect of natriuretic peptide-guided therapy on hospitalization or cardiovascular mortality in high-risk patients with heart failure and reduced ejection fraction: a randomized clinical trial. JAMA 318(8), 713-720 (2017).

12. Komajda M, Isnard R, Cohen-Solal A et al. Effect of ivabradine in patients with heart failure with preserved ejection fraction: the EDIFY randomised placebo-controlled trial. Eur. J. Heart Fail. 19(11), 1495-1503 (2017).

13. Feldman T, Mauri L, Kahwash R et al. A transcatheter interatrial shunt device for the treatment of heart failure with preserved ejection fraction (REDUCE LAP-HF I): a Phase II, randomized, sham-controlled trial. Circulation 137(6), 364-375 (2017).

14. Zinman B, Wanner C, Lachin JM et al. Empagliflozin, cardiovascular outcomes, and mortality in Type 2 diabetes. N. Engl. J. Med. 373(22), 2117-2128 (2015).

15. Neal B, Perkovic V, Mahaffey K et al. Canagliflozin and cardiovascular and renal events in Type 2 diabetes. N. Engl. J. Med. 377(7), 644-657 (2017).

16. Marrouche NF, Brachmann J, Andresen D et al. Catheter ablation for atrial fibrillation with heart failure. N. Engl. J. Med. 378(5), 417-427 (2018).

17. Zuchinali P, Souza GC, Pimentel $M$ et al. Short-term effects of high-dose caffeine on cardiac arrhythmias in patients with heart failure: a randomized clinical trial. JAMA Intern. Med. 176(12), 1752-1759 (2016).

18. Conrad N, Judge A, Tran J et al. Temporal trends and patterns in heart failure incidence: a population-based study of 4 million individuals. Lancet 391, 572-580 (2018). 